\title{
Public exposure formed by atmospheric discharges of nuclear power plants in ukraine
}

\author{
I. Bonchuk \\ Ukrainian Radiation Protection Institute, Kyiv 04050, 53, Melnikova str., Ukraine, \\ e-mail: bonchuk@rpi.kiev.ua
}

\begin{abstract}
Ukrainian Radiation Protection Institute has developed system for calculation of doses to the population living in the supervision zone of the NPP from atmospheric discharges of Nuclear Power Plants during normal operation. The system analyses daily meteorological data, and also daily and monthly atmospheric discharges of radionuclides. The developed system is applied for dose calculation for Ukrainian population and identification of radionuclides, which form considerable contribution to total doses (noble gases, H-3, I-131). A special attention has been given for variability of actual meteorological conditions and discharges. For example, it has been revealed the case when I-131 discharge from a Ukrainian NPP during 4 consecutive days has made $34 \%$ of annual discharge (and $72 \%$ during 3 weeks). Variability of actual discharges is a result of changes in an operation mode of NPP unit and it is a typical situation for Ukrainian NPPs. Apparently, the similar situation can be typical for NPPs in another countries. Such results lead to necessity to consider possible variations of meteorological conditions and discharges for establishment of permissible levels (for example, by introduction of variability factor).
\end{abstract}

\section{INTRODUCTION}

The problem of the analysis of the dosimetric conditions formed by atmospheric discharges of Nuclear Power Plants is an actual problem for the nuclear power engineering. This problem is important for emergency situations and radiation accidents as well as for the normal operation, because it is closely connected with establishing of permissible levels. The decision of this problem is related to complexities of the analysis and processing of a large amount of dynamically changing input data and presence of fallouts on the soil formed for a long time of NPP operation. The specified reasons cause complexity of prognosis of result and lead to disproportion of changes of population doses to discharge changes.

The paper contains results of the analysis of dosimetric conditions for the population living in a supervision zone of the Rivne NPP.

\section{METHODS FOR CALCULATION OF DOSES}

\subsection{Initial data}

For calculation of doses the initial data subdivided into two groups. The first group includes "dynamic" time-varying data, namely the meteorological data received from a meteorological station, operated by Rivne NPP, and values of atmospheric discharge for each controlled radionuclide, estimated at discharge stacks. Another group includes time-constant parameters ("static" data) which are specific for Rivne NPP. The second group incorporates the heights of discharge sources, distances from discharge sources to the border of a sanitary-protective zone (SPZ), etc.

Methods for calculation of doses for population living in the SPZ of Rivne NPP are focused first of all on possibilities of formation of the "dynamic" data about weather conditions and amount of discharge. 
The Radiation Safety Shop of Rivne NPP has a possibility to give a summary about following daily meteorological data:

- stability class of atmosphere from A to G (by Pasquill);

- wind directions for sixteen sectors $\left(22,5^{\circ}\right.$ each);

- wind speeds for ten ranges (from 0 to $60 \mathrm{~m} \cdot \mathrm{s}^{-1}$ ).

Besides, Radiation Safety Shop of Rivne NPP also provides a summary for daily data about precipitation with conversion of their washout ability to absolute washout ability of a rain with intensity $1 \mathrm{~mm} \cdot \mathrm{h}^{-1}$.

Amount of discharges are given in two forms:

- daily discharges of I-131 and noble radioactive gases (NRG);

- monthly discharges of H-3, Cr-51, Mn-54, Fe-59, Co-58, Co-60, Sr-90, Zr-95, Nb-95, Ag-110 m, Cs-134, Cs-137.

As the meteorological data and the data about amount of discharges are structurally different, there were assumptions about uniformity of discharges within a period for summary (within a day for ${ }^{131} \mathrm{I}$ and NRG, and within a month for others).

\subsection{Calculations of doses}

The structure of the meteorological data forms the following approach to calculations of doses to the population. The supervision zone of Rivne NPP is divided into 16 sectors corresponding to 16 wind directions in the meteorological data. Each sector is subdivided on subsectors (by distance from the discharge source). The subdividing by distance from the discharge source is made from $2.5 \mathrm{~km}$ (radius of SPZ of Rivne NPP) to $10 \mathrm{~km}$ for each sector (up to 20 "elements" in each sector). Thereby, each subsector is identified by coordinates $(\varphi, x)$, where $\varphi$ is an angle corresponding to the sector, and $x$ is a distance from the discharge source. Such subsector is called "subsector $(\varphi, x)$ ", and a whole sector is a "sector $\varphi$ ".

Calculations of doses for each subsector are carried out for the population of following reference age $(\tau)$ : 1 year, 5 years, 10 years, 15 years, Adult. All specified calculations are made for following pathways:

- external exposure from the radioactive cloud;

- external exposure from fallout on a soil;

- inhalation intake from radioactive cloud;

- ingestion with contaminated foodstuff.

The total annual effective dose to the population of reference age $\tau$ is defined by summation of all above specifies pathways for all considered radionuclides $(i)$ :

$$
E_{\tau}(\varphi, x)=\sum_{i}\left[E_{\tau, i}^{c l}(\varphi, x)+E_{\tau, i}^{s r f}(\varphi, x)+E_{\tau, i}^{\text {inhal }}(\varphi, x)+E_{\tau, i}^{\text {ingest }}(\varphi, x)\right] .
$$

All specified doses for a calendar year are determined by summation of corresponding 'daily' doses during all calendar dates of year $(\Theta)$. Effective doses of an external exposure to the population of reference age $\tau$ in subsector $(\varphi, x)$, formed during a calendar year by radionuclide $i$ in a cloud and in fallouts on a soil, are calculated, respectively:

$$
E_{\tau, i}^{c l}(\varphi, x)=k_{r, i} g_{\tau, i}^{c l} \sum_{\Theta} A_{V, i}(\varphi, x, \Theta), \quad E_{\tau, i}^{s r f}(\varphi, x)=k_{r, i} g_{\tau, i}^{s r f} \sum_{\Theta} \frac{A_{S, i}(\varphi, x, \Theta)}{L},
$$

where $k_{r, i}$ is a shielding factor (a behaviour mode) for radionuclide $i$;

$A_{V, i}(\varphi, x, \Theta)$ and $A_{S, i}(\varphi, x, \Theta)$ are daily average (for calendar date $\Theta$ ) volume (in a surface air) and surface (on a soil) specific activities of radionuclide $i$ in subsector $(\varphi, x)$;

$g_{\tau, i}^{c l}$ is an effective dose of an external exposure per unit of volume specific activity of radionuclide $i$ in air [1]; 
$g_{\tau, i}^{s r f}$ is an effective dose of an external exposure per unit of volume specific activity of radionuclide $i$ in the $5 \mathrm{~cm}$ layer of soil [1];

$L$ is a thickness of the top layer of soil $(5 \mathrm{~cm})$.

The shielding factor reflects exposure peculiarities for the population and reduces the doses. Considering the data [2], the following values of this factor are applied: 0.29 (for rural settlements), 0.2 (for urban villages and small towns with prevalence of one-storeyed buildings) and 0.13 (for cities).

Expected effective doses of the internal exposure to the population in subsector $(\varphi, x)$ because of inhalation and ingestion intakes of radionuclides during a calendar year are determined as follows:

$$
E_{\tau, i}^{\text {inhal }}(\varphi, x)=g_{\tau, i}^{\text {inhal }} \sum_{\Theta} A_{V, i}(\varphi, x, \Theta), \quad E_{\tau, i}^{\text {ingest }}(\varphi, x)=e_{\tau, i}^{\text {ingest }} \sum_{\Theta} I_{\tau, i}^{\text {ingest }}(\varphi, x, \Theta),
$$

where $g_{\tau, i}^{\text {inhal }}$ is an effective internal dose due to inhalation intake per unit of volume specific activity of radionuclide in air;

$e_{\tau, i}^{\text {ingest }}$ is an effective internal dose per unit of ingestion intake of radionuclide;

$I_{\tau, i}^{\text {ingest }}(\varphi, x, \Theta)$ is a daily ingestion intake of radionuclide.

Values $g_{\tau, i}^{\text {inhal }}$ and $e_{\tau, i}^{\text {ingest }}$ are calculated according to biokinetic and dosimetric models of the International Commission on Radiological Protection, in particular [3, 4].

As appears from the equations above, the base values for dose assessments are daily average volume (in air) and surface (in soil) specific activities of radionuclides. The Gaussian model for dispersion is applied to the calculations of the specified values [5, 6].

Daily average volume activity of radionuclide in a ground air in subsector $(\varphi, x)$ for calendar date $\Theta$ is determined by equation:

$$
A_{V, i}(\varphi, x, \Theta)=Q_{i}(\Theta) \frac{N}{\sqrt{2 \pi^{3} x}} \sum_{\chi}\left[\frac{1}{\sigma_{z, \chi}(x)} \exp \left(-\frac{h^{2}}{2 \sigma_{z, \chi}^{2}(x)}\right) \sum_{w} \frac{P_{\varphi, \chi, w}^{\Theta} F_{i, \chi, w}(\varphi, x, \Theta)}{u_{\varphi, \chi, w}^{\Theta}}\right]
$$

where $Q_{i}(\Theta)$ is a daily discharge of radionuclide;

$N$ is a number of sectors (16);

$\chi$ is a stability class of atmosphere;

$w$ is a wind speed range for a stability class $\chi$;

$P_{\varphi, \chi, w}^{\Theta}$ is a daily frequency of a stability class $\chi$ for a wind speed range $w$ in sector $\varphi$;

$\sigma_{z, \chi}(x)$ is a standard deviation in a vertical direction for a stability class $\chi$;

$u_{\varphi, \chi, w}^{\Theta}$ is a wind speed for a wind speed range $w$ in sector $\varphi$ for a stability class $\chi$;

$h$ is an effective discharge height;

$F_{i, \chi, w}(\varphi, x, \Theta)$ is an exhaustion function for radionuclide $i$ in a cloud for a stability class $\chi$ for a wind speed range $w$ in subsector $(\varphi, x)$ (it is a product of exhaustion functions because of dry deposition, wet deposition and radioactive decay).

Calculations of $\sigma_{z, \gamma}(x)$ and $F_{i, \gamma, w}(\varphi, x, \Theta)$ are carried out according to [5, 6].

Daily average surface activity of radionuclide on soil in subsector $(\varphi, x)$ for calendar date $\Theta$ is calculated by integration for all operating time of Rivne NPP:

$$
A_{S, i}(\varphi, x, \Theta)=\int_{\Theta_{0}}^{\Theta} \dot{A}_{S, i}(\varphi, x, t) \exp \left(-\Lambda_{\text {sum }, i}(\Theta-t)\right) d t
$$

where $\Theta_{0}$ is a beginning of fallout accumulation (commissioning of Rivne NPP);

$\dot{A}_{S, i}$ is a deposition rate of radionuclide on soil;

$\Lambda_{\text {sum }, i}$ is a constant of decrease of radionuclide activity; it is a sum of a constant of radioactive decay, rate of moving beyond the top (root) layer of soil and rate of fixation in soil. 
Table 1. The contribution to the annual effective doses (from 2008 to 2010) to the population living in the supervision zone of Rivne NPP $(\%)$.

\begin{tabular}{|l|c|c|c|c|c|c|}
\hline \multirow{2}{*}{$\begin{array}{l}\text { Radionuclide and } \\
\text { pathway }\end{array}$} & \multicolumn{3}{|c|}{ Children (1 y) } & \multicolumn{3}{c|}{ Adults } \\
\cline { 2 - 7 } & 2008 & 2009 & 2010 & 2008 & 2009 & 2010 \\
\hline NRG (cloud) & 41 & 40 & 29 & 48 & 39 & 48 \\
\hline H-3 (inhalation) & 30 & 49 & 20 & 39 & 53 & 37 \\
\hline I-131 (ingestion) & 24 & 7.3 & 46 & 3.6 & 0.9 & 5.7 \\
\hline Cs-137 (ingestion) & 0.9 & 0.8 & 0.7 & 3.4 & 2.6 & 3.7 \\
\hline Co-60 (soil) & 2.2 & 1.8 & 1.4 & 2.6 & 1.8 & 2.4 \\
\hline Cs-137 (soil) & 1.0 & 0.9 & 0.8 & 1.2 & 0.9 & 1.3 \\
\hline Others & 1.1 & 0.8 & 1.5 & 1.6 & 1.2 & 2.2 \\
\hline
\end{tabular}

Daily deposition rate of radionuclide on soil is estimated by summation of daily deposition rates of radionuclide on a soil surface in subsector $(\varphi, x)$ due to dry and wet depositions.

The important component of dosimetric calculations is a determination of ingestion intake of radionuclides. For this purpose ECOSYS model [7] was modified considering consumption levels of foodstuff which are typical for the Ukrainian population. As ingestion intakes of radionuclides with contaminated foodstuff essentially depend on seasonality of fallout, therefore calculations of daily intakes formed by fallouts in each separate day of year were performed according to the model. Such preliminary prepared sets were calculated for all reference ages.

As the detailed initial data (as described above) is accessible only since 2006, the average meteorological data and the data about discharge were used for the taking into account of consequences of discharges of Rivne NPP during previous years (formed the contamination of soil and the subsequent intake with a foodstuff).

\section{RESULTS AND DISCUSSION}

The data about weather conditions and amount of atmospheric discharges of Rivne NPP in 2006-2010 were used for calculations of doses.

By results of calculations (the Table 1) the highest annual doses are received by children (reference age 1 year). The main contribution to the total dose is formed by NRG, H-3, and I-131; contributions of Cs-137 and Co-60 are substantially smaller. Other radionuclides can be characterized as "negligibly small". I-131 almost completely forms a dose due to ingestion intake (first of all, consumption of milk and leafy vegetables). The contribution of I-131 to the annual effective dose is substantially higher for children than for adults ( $\sim 8$ times) due to the higher ingestion dose coefficient. Dominating pathway for NRG is an external exposure from the radioactive cloud, as well as an inhalation is for H-3.

The Figure 1 represents a variation of monthly atmospheric discharges of radionuclides presented in the Table 1. It is clear that a discharge of I-131 has a substantial non-uniformity. The ratio of the maximum monthly discharge to the minimum monthly discharge almost reaches 200 . The reason is that different numbers of power units were in different modes of operation during specified periods. The similar situation is observed also for others radionuclides, for example, for Co-58 this ratio equals to 208, for Ag-110m - to 174 , for Nb-95 - to 109 , for Cs-134 - to 90 . However, as opposed to I-131, the contribution of these radionuclides to the total dose is very small. Concerning radionuclides which can deserve attention (from the point of view of the contribution to the total dose) such ratio for Cs-137 equals to 64, and for Co-60 - to 53. The variations of discharge of NRG and H-3 are essentially lesser, they equal to 9.4 and 8.7 respectively.

Coming back to I-131, it is necessary to draw an attention that daily variability of its discharge is substantially higher than for monthly intervals. The mentioned ratio for daily discharge of I-131 is higher than 1100. For instance, discharge of I-131 during 4 consecutive days in September 2010 was 34\% of annual discharge (and during 3 weeks in August-Septemver 2010 it equals to 72\%). It 


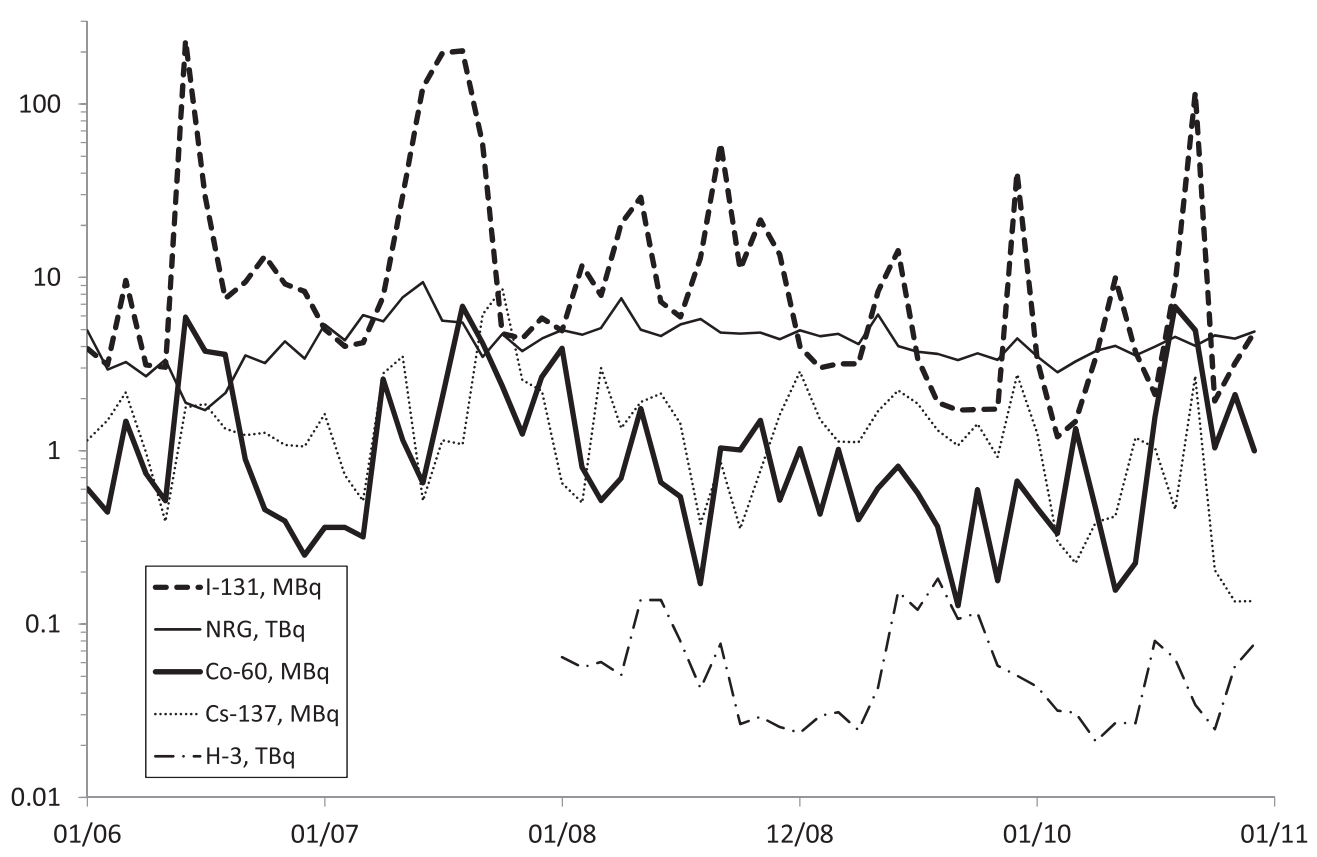

Figure 1. The variation of monthly atmospheric discharges of I-131, noble radioactive gases (NRG), Co-60, Cs-137, and $\mathrm{H}-3$.

Table 2. Maximal fractions of annual discharge of I-131 performed during continuous time interval (\%).

\begin{tabular}{|l|c|}
\hline $\begin{array}{l}\text { Time interval of } \\
\text { discharge }\end{array}$ & $\begin{array}{c}\text { Fraction of annual } \\
\text { discharge }\end{array}$ \\
\hline $1 \mathrm{~d}$ & 13.5 \\
\hline $3 \mathrm{~d}$ & 29 \\
\hline $7 \mathrm{~d}$ & 42 \\
\hline $10 \mathrm{~d}$ & 48 \\
\hline $15 \mathrm{~d}$ & 62 \\
\hline $3 \mathrm{wks}$ & 72 \\
\hline $1 \mathrm{mo}$. & 76 \\
\hline
\end{tabular}

is obvious, considering the small half-life time, I-131 is most "radiologically" dangerous in case of summer discharge. Detailed data about maximal fractions of annual discharge of I-131 produced during continuous time interval is provided in the Table 2.

The obtained results (especially for iodine) are important for regulation purposes, namely for the establishment of permissible levels of discharges.

The limits of discharges of radionuclides are calculated on the assumption of 'uniformity' of discharge amounts during a calendar year. At significant discharge of radionuclide during several consecutive days, the dispersion of radionuclides can occur over the same sectors, instead of annual averaged distribution over all sectors. Thus, the consideration of an actual weather conditions and actual discharges leads to essential differences from 'average' assessment. For example, an application of the average initial data results the annual doses from ingestion intake are almost 3 times less than for the actual data for Rivne NPP. Such results lead to necessity to consider a possible variation of initial data during procedures of establishment of discharge limits, for example, by introduction of variability factors. 


\section{CONCLUSION}

1. The main contribution to the annual effective doses to the population living in the supervision zone of Rivne NPP is formed by noble radioactive gases, H-3, and I-131. Their contribution is more than $90 \%$ for adults and $\sim 95 \%$ for children (1 year). The contribution of I-131 to the annual effective dose is substantially higher for children than for adults ( $\sim 8$ times) due to the higher ingestion dose coefficient.

2. Discharges of radionuclides (especially I-131) have essential variability throughout a year. The reason is that different numbers of power units are in different modes of operation during specified periods. Daily discharge of I-131 can be higher than 13\% from the annual discharge. Total discharge during 11 consecutive days can reach more than 50\% from the annual discharge, and it can be higher than $75 \%$ during 30 consecutive days. The doses which are calculated taking into account the actual meteorological data and dynamics of discharges almost 3 times higher the doses estimated for initial data uniformed during a year.

3. Essential variability of discharges leads to higher doses (in comparison with uniform discharges) and results to necessity of careful assessments of permissible levels. During the establishment of discharge limits it is reasonable to consider the observed variability of weather conditions and discharge amounts.

\section{References}

[1] K.F. Eckerman, R.W. Leggett, DCFPAK: Dose coefficient data file package for Sandia National Laboratory, Oak Ridge National Laboratory Report ORNL/TM-13347, Oak Ridge (1996).

[2] I. Likhtariov, L. Kovgan, D. Novak, S. Vavilov, P. Jacob, and H.G. Paretzke, Effective doses due to external irradiation from the Chernobyl accident for different population groups of Ukraine. Health Phys. 70(1) (1996).

[3] ICRP Publication 66, Human Respiratory Tract Model for Radiological Protection, Oxford: Pergamon Press (1994).

[4] ICRP Publication 67, Age-Dependent Doses to Members of the Public from Intake of Radionuclides: Part 2. Ingestion Dose Coefficients, Oxford: Pergamon Press (1993).

[5] IAEA, Atmospheric Dispersion in Nuclear Power Plant Siting. A Safety Guide. IAEA Safety series No. 50-SG-S3 (1980).

[6] IAEA, Generic Models and Parameters for Assessing the Environmental Transfer of Radionuclides from Routine Releases. Exposures of critical groups. IAEA Safety series N 57 (1982).

[7] H. Muller, G. Prohl, ECOSYS-87: A dynamic model for the assessment of the radiological consequences of nuclear accidents. Health Phys. 64 (1993). 